\title{
Recent Suicidal Ideation Among Patients in an Inner City Emergency Department
}

\author{
Mark A. Ilgen, PhD, Maureen A. Walton, PhD, Rebecca M. Cunningham, MD, \\ Kristen L. Barry, PhD, Steve T. Chermack, PhD, Peter De Chavez, MS, \\ and Frederic C. Blow, $\mathrm{PhD}$
}

The rates and associated features of suicidal ideation among 5,641 patients seeking routine, nonsuicide related care in an inner-city emergency department were examined. Approximately $8 \%$ of patients seeking routine care in the emergency department reported some form of suicidal ideation within the past 2 weeks. Suicidal ideation was common in individuals who were single with poorer mental health, had higher depression, and had received some drug or alcohol treatment in the past 3 months or had used cocaine or marijuana in the past 30 days. Improved screening procedures could help to identify routine care patients who are at risk for suicide.

On average, over 30,000 people die by suicide every year in the United States (Centers for Disease Control and Prevention [CDC], 2007). In additional, population surveys indicate that, at some point during their lifetimes, $5 \%$ of the U.S. population report a suicide attempt and $14 \%$ report serious suicidal ideation (Kessler, Borges, \& Walters, 1999).

Over 395,000 U.S. emergency depart- ment (ED) visits occurred in 2006 for the treatment of nonfatal intentional self-injuries (CDC, 2007), and recent estimates indicate that approximately $40 \%$ of patients who died by suicide were seen in an emergency department within 12 months of death (Gairin, House, \& Owens, 2003). Thus, the emergency department is an important setting for suicide risk assessment and intervention efforts (Healy, Barry, Blow, Welsh, \& Milner,

Mark Ilgen, Kristen Barry, Steve Chermack, and Federic Blow are with the Department of Veterans Affairs, Health Services Research \& Development in Ann Arbor, MI, and the Department of Psychiatry at the University of Michigan, Ann Arbor; Maureen Walton and Peter De Chaves are affiliated with the Department of Psychiatry at the University of Michigan, Ann Arbor; and Rebecca Cunningham is with the Department of Emergency Medicine at the University of Michigan, Ann Arbor.

This work was supported by the Department of Veterans Affairs Health Services Research (HSR\&D) grant MRP 05-137 and by grants from the National Institute on Alcohol Abuse and Alcoholism (NIAAA; \#AA014665) and the National Institute on Drug Abuse (NIDA; \#DA 016591). The views expressed in this report are those of the authors and do not necessarily represent the views of the Department of Veterans Affairs, NIAAA, or NIDA. Additionally, neither the Department of Veterans Affairs, NIAAA, nor NIDA had any direct involvement in the study design; the collection, analysis, and interpretation of data; the writing of the report; or in the decision to submit the paper for publication. All authors declare that they have no conflicts of interest. We would like to thank the medical staff and patients at Hurley Medical Center for their support of this research. In addition, we would like to acknowledge the project coordinator, Lynn Massey, who was essential to the success of this research.

Address correspondence to Mark Ilgen, PhD, VA Serious Mental Illness Treatment Research and Evaluation Center, University of Michigan Department of Psychiatry, 2215 Fuller Road (11H), Ann Arbor, MI 48105; E-mail: marki@umich.edu 
2006; Institute of Medicine, 2002). More generally, the Emergency Department is an ideal location in which to implement screening efforts designed to treat traditionally underserved populations, such as in inner-city settings where patients may have less access to other sources of medical care (McCaig \& Burt, 2003). However, the vast majority of patients seen in an ED prior to the enactment of suicide are seen for reasons not explicitly related to suicidality (Gairin et al., 2003). Most research in suicide in relation to the ED is focused on individuals with explicit suicidality as a presenting problem at the time of treatment in an ED. Recent research indicates that over $10 \%$ of those seen in ED have suicidal ideation that is not reported to staff (Claassen \& Larkin, 2005). This study also found that suicidal ideation was more common in those with elevated depression, anxiety, and substance-related problems. However, more comprehensive data are needed to better understand the relative impact of differing risk factors for suicidal ideation in multivariate models. Because the level of severity of suicidality occurs along a continuum (Conner et al., 2007; Kessler et al., 1999), examining the level of suicidal ideation in patients who are seeking general care in an ED would expand the understanding of the prevalence and characteristics of these high risk patients (Buzan \& Weissberg, 1992).

In the present study, we documented the rate of recent (i.e., last 2 weeks) suicidal ideation in patients seen in a large inner-city ED for reasons other than acute suicidality. After documenting the rate of suicidal ideation in those not initially identified by ED staff as suicidal, we examined the relative associations among mental health, substance use, and suicidal ideation.

\section{MATERIALS AND METHODS}

\section{Study Design}

We used a cross-sectional examination of data obtained from adult ED patients who completed a brief self-report computerized screening survey that included items about the presence and severity of psychiatric symptoms, substance use, and suicidal ideation. The computerized screening survey was administered after written consent was obtained from eligible patients and took approximately 10 minutes to complete. The study was approved by the University of Michigan and the Hurley Medical Center Institutional Review Boards, and certificates of confidentiality were obtained from NIAAA and NIDA. The present study was part of a larger project that excluded all patients with diagnoses related to suicidality (for more details, see Blow et al., 2006).

\section{Study Setting and Population}

The study was conducted in an innercity, university-affiliated Level 1 ED (Hurley Medical Center in Flint, Michigan) with an annual census of approximately 75,000 patients. All study eligible (see below) adult patients presenting to the $\mathrm{ED}\left(9_{\mathrm{AM}}-11 \mathrm{PM}, 7\right.$ days per week) were recruited to complete a health screening survey during a 1-year period (April 2006-March 2007). It was decided to not recruit between $11 \mathrm{PM}$ and $9 \mathrm{AM}$ due to relatively low numbers of individuals presenting during this time period, resulting in low efficiency in terms of low subject recruitment given staff allocation.

\section{Study Protocol}

Patients between the ages of 19 and 60 were approached by research staff to participate. Patients were excluded from the study if they were pregnant, had unstable vital signs (triaged to the resuscitation bay), were unable to provide informed consent (e.g., unconscious, police custody, acutely intoxicated), or had presented for psychiatric evaluations (including acute suicidality). More specifically, determination of acute suicidality was based on a combination of the patient's chief complaint and the judgment of the clinical staff. If the patient's chief complaint was listed in the triage $\log$ as a suicide attempt or suicidal ideation, research staff did not approach the 
patient. If the chief complaint was listed as an overdose, the research assistant talked with ED staff to see if it was because of a suicide attempt. An indication of acute suicidality by either the patient or the clinical staff was sufficient for participants to be excluded from the current study.

After determination of study eligibility, participants consented and completed the screening survey. During the course of the screening survey, if research staff became aware of current suicidal ideation, they provided the participant with a list of community resources and encouraged the participant to talk to ED staff about their concerns. (Note that our consent procedures allow staff to breach confidentiality if a person is suicidal; however, in every case the participant agreed to talk with ED staff). During the ED visit, seven participants were excluded from the study after consenting and prior to completing the screening survey because they were acutely suicidal.

\section{Measurements}

Research assistants used a computerized survey specially programmed for the study to interview participants. This screening instrument was primarily composed of questions or subsections of broader, empirically validated instruments (specific details provided below). Questions about alcohol, drug use, and suicide were included in an overall brief health and lifestyle screening instrument to maximize the accuracy of responses and to put more sensitive questions in the broader context of health behaviors and health screening (Saunders, Aasland, Babor, Fuente, \& Grant, 1993). This instrument also contained questions about patient demographics, income level, and health insurance status.

Suicidal Ideation and Depression. The determination of the presence of suicidal ideation was based on a single item of the $\mathrm{Pa}$ tient Health Questionnaire (PHQ-9; Kroenke, Spitzer, \& Williams, 2001; Spitzer, Kroenke, \& Williams, 1999). Specifically, patients were asked how often in the previous 2 weeks they had, "Thoughts that you would be better off dead or of hurting yourself in some way." The PHQ-9 includes each of the nine DSM$I V$ criteria for depression (including suicidal ideation) coded as 0 (not at all) to 3 (nearly every day) for the previous 2 weeks. The PHQ-9 has $84 \%$ specificity and $95 \%$ sensitivity for current major depressive disorder in a primary care population (Löwe et al., 2004). Because of the high likelihood that ratings greater than 1 would be rare, and the anticipated skewed distribution of this item, it was recoded into a dichotomous indicator of the presence of any suicidal ideation ( $n o=0$ vs. $y e s=1)$ in the past 2 weeks. This item served as the primary dependent variable in the present study. Additionally, the other eight items of the PHQ-9 were used to measure severity of depression.

Psychosocial and Medical Functioning. The 12-item Short Form (SF-12) was used to assess physical functioning, social functioning, physical and emotional role functioning, mental health, general health perceptions, vitality, and pain (Ware, 1976; Ware, Kosinski, \& Keller, 1996; Ware \& Sherbourne, 1992). The SF-12 indicators of health status have been widely studied and implemented as standardized screening tools (Fleming, Barry, Manwell, Johnson, \& London, 1997; Wallace, Cutler, \& Haines, 1988). In the present study, we used a cut-off of lower than the 25 th percentile on the SF-12 indicators to measure low functioning in each domain.

Alcobol and Drug Use and Treatment. Recent substance use and related symptoms were determined by items from the Substance Abuse Outcomes Module (SAOM; Smith, Burnam, Mosley, Hollenberg, Mancino, \& Grimes, 2006). The items from the SAOM utilized in the present study measured past 30-day alcohol use, binge drinking, cocaine, marijuana, and tobacco use. The SAOM is widely used and has sound psychometric properties (Smith et al., 2006). Finally, all participants were asked whether or not they had received drug or alcohol treatment within the past 3 months. 


\section{Data Analysis}

All analyses were conducted with SAS version 9.1 for Windows. First, we documented the rate of suicidal ideation in this sample. Second, we examined individual bivariate associations between all demographic characteristics, aspects of functioning, and alcohol and drug use with suicidal ideation using a Chi-square test of independence for categorical predictors and a two-sample $t$ test for continuous predictors. Finally, we conducted a logistic regression analysis that included all factors that were significant in the previous bivariate analyses to examine the relative impact of each of these on risk of suicidal ideation.

\section{RESULTS}

A total of 8,756 potentially eligible patients presented to the ED during the study recruitment period; 1,455 (16.6\%) patients were missed (typically because the recruiters were busy with another patient). Of the 7,301 patients approached for participation in the study, 5,641 (77.3\%) consented to participate and 1,660 (22.7\%) refused (e.g., primarily too ill, tired or weak, or in too much pain to participate). Due to IRB consent restrictions, the only information retained for refusals was race and gender; $46 \%$ of the refusals were male and $57 \%$ were African American. No data are available on the extent to which participants recruited during study hours $\left(9_{\mathrm{AM}}\right.$ to $11 \mathrm{PM})$ reflect the patients typically seen between 11 PM and 9AM. Similarly, no data are available on the number of patients who were missed because they were too intoxicated or because they were determined to be in need of immediate psychiatric evaluation.

Approximately $42 \%(n=2,388)$ of participants were male, $56 \%(n=3,148)$ were African American, and 28\% $(n=1,584)$ reported that they were married or living with a partner. The mean age of the sample was $36.51(S D=11.6)$, and $34 \%(n=1,936)$ were between 19-29 years old. About one third
(35\%; $n=1,953)$ had some college education or higher. Also, the majority $(67 \%, n=2,753)$ had an annual income below $\$ 20,000$ and, during the time the survey was conducted, less than half $(47 \%, n=2,637)$ were employed either part or full time. On average, the reported physical and mental health component scores were $45.46(S D=11.4)$ and $46.18(S D=12.9)$, respectively, which were lower compared to mean scores of 50.12 and 50.04, respectively, from previously studied community samples (Ware, 1976; Ware \& Sherbourne, 1992).

\section{Rates and Associated Features of Suicidal Ideation}

Within the 2 weeks prior to presenting to the $\mathrm{ED}, 5 \%(305 / 5,641)$ of the sample reported suicidal ideation for several days, $1 \%$ $(51 / 5,641)$ reported suicidal ideation more than half of the days, and $2 \%(92 / 5,641)$ reported suicidal ideation nearly every day. Collapsing across these three responses, suicidal ideation within the past 2 weeks was reported in $8 \%(448 / 5,641)$ of the sample.

As presented in Table 1, recent suicidal thoughts were more common in patients who were not married or living with a partner $(p<0.0001)$; who had lower educational attainment $(p=0.0002)$, annual income $(p<0.0001)$, employment status $(p<0.0001)$, physical health functioning $(p<0.0001)$, and mental health functioning $(p<0.0001)$; and who had received treatment for drug or alcohol problems in the past 3 months $(p<0.0001)$. Additionally, patients who reported past 30day use of tobacco $(p<0.0001)$, alcohol $(p=$ $0.0280)$, cocaine $(p<0.0001)$, marijuana $(p<$ $0.0001)$, binge drinking $(p<0.0001)$, and more depressive symptoms $(p<0.0001)$ were more likely to report recent suicidal ideation. In this sample, suicidal ideation was not significantly associated with gender, age, racial background, or health insurance status.

Relative Impact of Patient Characteristics on Risk of Suicidal Ideation

The predictors identified as significant correlates of suicidal ideation in the previous 


\section{TABLE 1}

Bivariate Demographic, Substance Use, and Psychological Correlates of Suicide Thoughts $(\mathrm{N}=5,641)$

\begin{tabular}{|c|c|c|c|c|c|c|}
\hline \multirow[b]{2}{*}{ Variable } & \multicolumn{2}{|c|}{$\begin{array}{l}\text { No Suicidal } \\
\text { Thoughts } \\
(n=5,193)\end{array}$} & \multicolumn{2}{|c|}{$\begin{array}{l}\text { Suicidal } \\
\text { Thoughts }^{a} \\
(n=448)\end{array}$} & \multicolumn{2}{|c|}{$\begin{array}{c}\text { Total } \\
(n=5,641)\end{array}$} \\
\hline & $n$ & $(\%)$ & $n$ & (\%) & $N$ & $(\%)$ \\
\hline \multicolumn{7}{|l|}{ Gender } \\
\hline Male & 2,205 & (92.34) & 183 & (7.66) & 2,388 & $(42.33)$ \\
\hline Female & 2,988 & $(91.85)$ & 265 & $(8.15)$ & 3,253 & $(57.67)$ \\
\hline \multicolumn{7}{|l|}{ Age } \\
\hline 19-29 Years Old & 1,781 & (91.99) & 155 & $(8.01)$ & 1,936 & $(34.32)$ \\
\hline 30 Years Old and Above & 3,412 & (92.09) & 293 & (7.91) & 3,705 & $(65.68)$ \\
\hline \multicolumn{7}{|l|}{ Race } \\
\hline Black of African American & 2,909 & $(92.41)$ & 239 & (7.59) & 3,148 & $(55.81)$ \\
\hline Not Black nor African American & 2,284 & $(91.62)$ & 209 & (8.38) & 2,493 & $(44.19)$ \\
\hline \multicolumn{7}{|l|}{ Marital Status* } \\
\hline Married or Living Together & 1,503 & (94.89) & 81 & $(5.11)$ & 1,584 & $(28.08)$ \\
\hline Never Married, Divorced, Separated, or Widowed & 3,690 & $(90.95)$ & 367 & $(9.05)$ & 4,057 & (71.92) \\
\hline \multicolumn{7}{|l|}{ Education** } \\
\hline Some College or College Grad & 1,834 & $(93.91)$ & 119 & $(6.09)$ & 1,953 & $(34.62)$ \\
\hline High School Grad or Less & 3,359 & $(91.08)$ & 329 & $(8.92)$ & 3,688 & $(65.38)$ \\
\hline \multicolumn{7}{|l|}{ Health Insurance ${ }^{\mathrm{b}}$} \\
\hline With Insurance & 4,038 & $(91.81)$ & 360 & $(8.19)$ & 4,398 & (78.16) \\
\hline No Insurance & 1,143 & $(93.00)$ & 86 & $(7.00)$ & 1,229 & $(21.84)$ \\
\hline \multicolumn{7}{|l|}{ Annual Income $e^{*, b}$} \\
\hline Less than $\$ 20,000$ & 2,453 & $(89.10)$ & 300 & $(10.90)$ & 2,753 & $(66.82)$ \\
\hline$\$ 20,000$ and Above & 1,312 & (95.98) & 55 & $(4.02)$ & 1,367 & (33.18) \\
\hline \multicolumn{7}{|l|}{ Employment Status, ${ }^{\star,}$} \\
\hline Employed & 2,516 & $(95.41)$ & 121 & $(4.59)$ & 2,637 & $(46.81)$ \\
\hline Not Employed & 2,670 & $(89.09)$ & 327 & $(10.91)$ & 2,997 & $(53.19)$ \\
\hline \multicolumn{7}{|l|}{ SF12 Physical Component* } \\
\hline Less Than or Equal to 25th Percentile & 1,236 & $(87.60)$ & 175 & $(12.40)$ & 1,411 & $(25.01)$ \\
\hline Above 25th Percentile & 3,957 & $(93.55)$ & 273 & $(6.45)$ & 4,230 & $(74.99)$ \\
\hline \multicolumn{7}{|l|}{ SF12 Mental Component* } \\
\hline Less Than or Equal to 25th Percentile & 1,084 & $(76.82)$ & 327 & (23.18) & 1,411 & $(25.01)$ \\
\hline Above 25th Percentile & 4,109 & (97.14) & 121 & $(2.86)$ & 4,230 & (74.99) \\
\hline \multicolumn{7}{|l|}{ Substance Abuse Treatment* ${ }^{*}$} \\
\hline Had Treatment in the Past 3 Months & 211 & $(78.73)$ & 57 & $(21.27)$ & 268 & $(4.75)$ \\
\hline No & 4,982 & $(92.72)$ & 391 & $(7.28)$ & 5,373 & $(95.25)$ \\
\hline \multicolumn{7}{|l|}{ Tobacco Use* } \\
\hline Used Tobacco in the past 30 Days & 2,712 & $(89.71)$ & 311 & $(10.29)$ & 3,023 & $(53.59)$ \\
\hline No & 2,481 & $(94.77)$ & 137 & $(5.23)$ & 2,618 & $(46.41)$ \\
\hline \multicolumn{7}{|l|}{ Alcohol Use ${ }^{\star * *}$} \\
\hline Had Alcohol in the Past 30 Days & 2,317 & (91.18) & 224 & $(8.82)$ & 2,541 & $(45.05)$ \\
\hline No & 2,876 & $(92.77)$ & 224 & (7.23) & 3,100 & $(54.95)$ \\
\hline \multicolumn{7}{|l|}{ Binge Drinking* } \\
\hline Binge Drinker in the Past 30 Days & 1,077 & $(89.23)$ & 130 & $(10.77)$ & 1,207 & $(21.40)$ \\
\hline No & 4,116 & $(92.83)$ & 318 & $(7.17)$ & 4,434 & $(78.60)$ \\
\hline \multicolumn{7}{|l|}{ Cocaine Use* } \\
\hline Used Cocaine in the Past 30 Days & 180 & $(72.29)$ & 69 & $(27.71)$ & 249 & $(4.41)$ \\
\hline No & 5,013 & $(92.97)$ & 379 & $(7.03)$ & 5,392 & $(95.59)$ \\
\hline
\end{tabular}


TABLE 1

Continued

\begin{tabular}{|c|c|c|c|c|c|c|}
\hline \multirow[b]{2}{*}{ Variable } & \multicolumn{2}{|c|}{$\begin{array}{c}\text { No Suicidal } \\
\text { Thoughts } \\
(n=5,193)\end{array}$} & \multicolumn{2}{|c|}{$\begin{array}{l}\text { Suicidal } \\
\text { Thoughts }^{\mathrm{a}} \\
(n=448)\end{array}$} & \multicolumn{2}{|c|}{$\begin{array}{c}\text { Total } \\
(n=5,641)\end{array}$} \\
\hline & $n$ & $(\%)$ & $n$ & $(\%)$ & $N$ & $(\%)$ \\
\hline \multicolumn{7}{|l|}{ Marijuana Use* } \\
\hline Used Marijuana in the Past 30 Days & 966 & $(87.11)$ & 143 & $(12.89)$ & 1,109 & $(19.66)$ \\
\hline No & 4,227 & $(93.27)$ & 305 & $(6.73)$ & 4,532 & $(80.34)$ \\
\hline Other Variables & Mean & $( \pm \mathrm{SD})$ & Mean & $( \pm \mathrm{SD})$ & Mean & $( \pm \mathrm{SD})$ \\
\hline Age & 36.55 & $(11.58)$ & 36.09 & $(11.13)$ & 36.51 & $(11.55)$ \\
\hline Depression Score (PHQ Total; 8 Items) ${ }^{*}$ & 5.13 & $(5.20)$ & 13.63 & $(5.90)$ & 5.81 & $(5.74)$ \\
\hline
\end{tabular}

${ }^{\mathrm{a}}$ In prior 2 weeks

bome subjects did not respond or skipped this question

${ }^{*} p<0.0001 ;{ }^{* *} p<0.001 ;{ }^{* * *} p<0.05$

analyses were entered into a logistic regression analysis to examine the relative impact of each factor on suicidal ideation (see Table 2). Individuals with suicidal thoughts were less likely to be married or living with a partner $(\mathrm{OR}=0.69)$, more likely to have a low mental health score $(\mathrm{OR}=2.54)$, more likely to have a higher depression score $(\mathrm{OR}=1.18)$, more likely to have received addictions treatment in the prior 3 months $(\mathrm{OR}=2.27)$, and more likely to have used cocaine or marijuana in the past 30 days $(\mathrm{OR}=1.82$ and $\mathrm{OR}=1.40$, respectively).

\section{DISCUSSION}

Recent suicidal ideation is common in individuals seen in an inner-city ED setting, with approximately $8 \%$ of patients presenting to the ED for a nonsuicide related injury or medical complaint reporting some form of suicidal ideation within the past 2 weeks. This rate of suicidal ideation is generally consistent with the rates that were described by Claassen and Larkin (2005) in their examination of occult suicidality among ED patients, and more than twice as high as the estimated prevalence $(3.3 \%)$ of 12 -month suicidal ideation in the general U.S. popula- tion (Kessler, Berglund, Borges, Nock, \& Wang, 2005). Within this ED sample, suicidal ideation was particularly common in those individuals who were single, had poorer mental health, had more depressive symptoms, had treatment for alcohol or drug problems in the past 3 months, or had used cocaine or marijuana in the past 30 days. This study is one of the first to measure the rate of suicidal ideation in patients seeking treatment for nonsuicide-related injury or medical complaint in an urban ED.

Based on the examination of patient treatment records, previous research indicates that approximately $.4 \%$ of all $\mathrm{ED}$ visits are for treatment of attempted suicide and self-inflicted injury (Doshi, Boudreaux, Wang, Pelletier, \& Camargo, 2005). The present findings indicate that, in addition to those explicitly presenting for management of acute suicide attempt or specifically requesting treatment for thoughts of self-harm, a high number of patients seeking treatment for other reasons also report high rates of recent suicidal ideation. As noted previously, this is the second study to document a substantial number of individuals in the ED with suicidal ideation who do not report this to the clinical staff. Both the present study and that of Claassen and Larkin (2005) have broadly similar 
TABLE 2

Multivariate Logistic Regression Analysis for Suicidal Thoughts by Demographics, Health Status, and Substance Use

\begin{tabular}{lccc}
\hline & \multicolumn{3}{c}{ Suicidal Thoughs $^{\mathrm{a}}$} \\
\cline { 2 - 4 } Variables & OR & \multicolumn{2}{c}{$95 \%$ CI } \\
\hline Married or Living Together* & 0.689 & 0.522 & 0.909 \\
Some College or College Grad & 0.803 & 0.624 & 1.034 \\
Employed & 0.810 & 0.627 & 1.047 \\
SF12 Physical Comp $\leq 25$ th Percentile & 1.043 & 0.817 & 1.332 \\
SF12 Mental Comp $\leq 25$ th Percentile* & 2.541 & 1.939 & 3.330 \\
Addictions Treatment in the Past 3 Months* & 2.268 & 1.540 & 3.341 \\
Used Tobacco in the Past 30 Days & 1.197 & 0.931 & 1.539 \\
Alcohol Use in the Past 30 Days & 1.107 & 0.824 & 1.489 \\
Binge Drinker in the Past 30 Days & 1.061 & 0.766 & 1.471 \\
Cocaine use in the Past 30 Days* & 1.824 & 1.239 & 2.686 \\
Marijuana Use in the Past 30 Days* & 1.396 & 1.069 & 1.823 \\
Depression Score (PHQ Total; 8 Items)* & 1.184 & 1.159 & 1.209 \\
\hline
\end{tabular}

${ }^{\mathrm{a}}$ In prior 2 weeks

* $p<0.05$

designs. However, the similarity in rates $(8 \%$ vs. $10 \%$ ) between these two studies is striking given the differences in geographic location (Michigan vs. Texas), demographic characteristics of the two samples, and sampling frame used to survey ED patients.

Prior work has identified previous seeking of ED care for acute suicidal ideation as one of the strongest predictors of subsequent suicide mortality (Crandall, FullertonGleason, Aguero, \& LaValley, 2006). Crandall et al. found that those with suicidal ideation documented in their medical chart at the time of ED treatment were over 6.5 times more likely to ultimately die by suicide than those without documented suicidal ideation. Buzan and Weissberg (1992) split potentially suicidal patients seen in the ED into those with "overt" and "covert" suicidality. These authors emphasized the fact that attending only to those with overt suicidality misses many potentially high risk patients. The present results highlight the existence of a large group of patients at elevated risk for suicide who do not report concerns about suicide to ED staff.

Given the high rate of suicide attempts and recent suicidal ideation, the ED is clearly an appropriate setting for the identification of patients at high risk for suicide (Baraff, Janowicz, \& Asarnow, 2006; Institute of Medicine, 2002; Kapur, Cooper, Hiroeh, May, Appleby, \& House, 2004). These efforts are particularly important in inner-city settings, where the ED often serves as primary care. For example, data from the National Hospital Ambulatory Medical Care Survey indicated an overall $20 \%$ increase in ED utilization between 1992-2001 (especially among African Americans, whose utilization rate was $76 \%$ higher than Whites) in concert with a $15 \%$ decrease in the number of ED nationally (McCaig \& Burt, 2003). Thus, implementing screening efforts in the ED can identify high risk patients who may not receive care in other settings. Once identified, recent evidence indicates that targeted psychosocial interventions are effective at reducing subsequent suicidal behaviors in high risk patients recruited from an ED (Brown, Ten Have, Henriques, Xie, Hollander, \& Beck, 2005).

Screening a large volume of patients in the ED is logistically challenging and no 
consensus exists for the best possible methods for identifying at-risk individuals. However, combining a positive computerized screening with a more comprehensive interview could partially offset some of the staff demands. For example, the screening items from the Beck Scale for Suicidal Ideation (BSS; Beck \& Steer, 1991) could be modified to the computerized format that was utilized in the present study. Screening positive for recent suicidal ideation, plans, or attempts on this computerized screen could trigger a more comprehensive clinical interview conducted by staff with mental health training (Cochrane-Brink, Lofchy, \& Sakinofsky, 2000). Although this would require additional resources on the part of the ED, it is arguable that this increased level of clinical attention is warranted given the potential to meaningfully detect and intervene with individuals at risk for suicide.

A unique strength of the present study was the use of multivariate models to examine the relative strength of the association among sociodemographic characteristics, psychiatric symptoms, and suicidal ideation. Consistent with prior research (Claassen \& Larkin, 2005; Doshi et al., 2005), patients with recent suicidal ideation were significantly more likely to report elevated depression and lower mental health functioning. Additionally, patients with recent cocaine and marijuana use and drug and alcohol treatment were more likely to report suicidal ideation even after accounting for depression and other measures of poor mental health. Past research has consistently linked recent drug and alcohol use to increased risk for suicidality in the ED (e.g., Claassen \& Larkin, 2005; Doshi et al., 2005); however, it was unclear from prior findings whether or not drug and alcohol problems represented risk factors for suicide after accounting for depression. The present results indicate that recent cocaine and marijuana use may be unique and separate indicators of heightened risk. Other research on nonclinical samples has consistently found an association between marijuana dependence and elevated risk for suicidal thoughts and behaviors (e.g., Lynskey et al., 2004). Even in more severe samples of individuals seeking treatment for drug and alcohol problems, the use of cocaine increases the risk of subsequent suicidal behaviors (Ilgen, Harris, Moos, \& Tiet, 2007). The association between substance use and suicidal thoughts in the ED likely reflects a combination of factors including negative affect, hopelessness, impulsivity, negative life events, and interpersonal difficulties in those with substance use disorders (Conner \& Duberstein, 2004).

Although previous studies have identified race as a predictor of suicidality (Doshi et al., 2005), race was not significantly associated with suicidal ideation in the present study. This could possibly reflect the fact that a large number of participants of all ethnic and racial groups in the present inner-city ED sample were from disadvantaged backgrounds, thus minimizing the differences between such groups.

\section{Limitations}

The present findings should be interpreted with caution for several reasons. First, although the sampling method was designed to accurately reflect the patients seen at a large, university affiliated, inner-city ED, this sample was drawn from a single site and the extent to which these findings generalize to other settings is unknown. This may be particularly true of EDs in different regions of the country or those serving populations with a different ethnic or racial composition. Unfortunately, no data are available on the total number of patients deemed to be acutely suicidal in this ED and the exclusion of these individuals almost certainly resulted in an underestimate in the overall rate of suicidal ideation in all patients seen in the ED. However, the group of patients specifically seeking care for acute management of suicide gesture or ideation has been well-studied in other samples. Future research is needed to establish the rate of suicidal ideation in all ED patients.

All analyses are cross-sectional. Thus, the extent to which variables of interest such 
as depression or substance use caused elevated suicidal ideation is unknown. The sample included only ED patients who were seen between 11PM and 9 $\mathrm{AM}$ and may not be representative of those patients seen during the overnight hours. Additionally, although the assessment of suicidal ideation was based on a single item whose reliability and validity has not been established, this item is part of a standardized questionnaire with acceptable psychometric properties. No other data were available about the extent of current suicidal intent or planning, nor do we have data on prior suicidal thoughts or behaviors in this sample. Nevertheless, suicidal ideation was measured close to the time of ED treatment and likely detects a group of patients at elevated, proximal risk for suicide. Also, other areas of interest (such as depression and alcohol use) were assessed using brief self-report measures. The use of abbreviated measures allowed us to assess a large sample, but future work is needed to establish the extent to

\section{REFERENCES}

Baraff, L. J., Janowicz, N., \& Asarnow, J. R. (2006). Survey of California emergency departments about practices for management of suicidal patients and resources available for their care. Annals of Emergency Medicine, 48, 452-458.

Beck, A. T., \& Steer, R. A. (1991). Manual for Beck Scale for Suicidal Ideation. New York: Psychological Corporation.

Blow, F. C., Barry, K. L., Walton, M. A., Maio, R. F., Chermack, S. T., Bingham, C. R., eT AL. (2006). The efficacy of two brief intervention strategies among injured, at-risk drinkers in the emergency department: Impact of tailored messaging and brief advice. The Fournal of Studies on Alcohol, 67, 568-578.

Brown, G. K., Ten Have, T., Henriques, G. R., Xie, S. X., Hollander, J. E., \& Beck, A. T. (2005). Cognitive therapy for the prevention of suicide attempts: A randomized controlled trial. The Journal of the American Medical Association, 294, 563-570.

Buzan, R. D., \& Weissberg, M. P. (1992). Suicide: Risk factors and therapeutic considerations in the emergency department. The Fournal of Emergency Medicine, 10, 335-343.

Centers for Disease Control and Prevention. (2007). Web-based injury statistics query which these measures reflect other gold standard measures of psychiatric and substancerelated problems in this population.

\section{Conclusion}

Despite the above limitations, this study broadens our knowledge of the rates and associated features of ideation among patients not explicitly treated for suicidality in ED settings. These findings suggest that many patients who do not initially report a suicide attempt have had significant suicidal thoughts within the past 2 weeks. Suicidal thoughts are most common in single patients with poorer mental health, more depressive symptoms, who have had some drug and alcohol treatment in the past 3 months, or those who have used cocaine or marijuana in the past 30 days. In order to identify patients at high risk for suicide seen in ED, expanding screening efforts beyond those explicitly treated for suicidality should be considered.

and reporting system: Leading causes of death reports. Atlanta, GA: Author.

Claassen, C. A., \& Larkin, G. L. (2005). Occult suicidality in an emergency department population. The British Fournal of Psychiatry, 186, 352-353.

Cochrane-Brink, K. A., Lofchy, J. S., \& SAKinofsky, I. (2000). Clinical rating scales in suicide risk assessment. General Hospital Psychiatry, 22, 445-451.

Conner, K. R., \& Duberstein, P. R. (2004). Predisposing and precipitating factors for suicide among alcoholics: Empirical review and conceptual integration. Alcoholism, Clinical and Experimental Research, 28, 6S-17S.

Conner, K. R., Hesselbrock, V. M., Meldrum, S. C., Schuckit, M. A., Bucholz, K. K., Gamble, S. A., et al. (2007). Transitions to, and correlates of, suicidal ideation, plans, and unplanned and planned suicide attempts among 3,729 men and women with alcohol dependence. Journal of Studies on Alcohol and Drugs, 68, 654662.

Crandall, C., Fullerton-Gleason, L., Aguero, R., \& LaValley, J. (2006). Subsequent suicide mortality among emergency department patients seen for suicidal behavior. Academic Emergency Medicine, 13, 435-442. 
Doshi, A., Boudreaux, E. D., Wang, N., Pelletier, A. J., \& Camargo Jr., C. A. (2005). National study of U.S. emergency department visits for attempted suicide and self-inflicted injury, 1997-2001. Annals of Emergency Medicine, 46, 369375.

Fleming, M. F., Barry, K. L., Manwell, L. B., Johnson, K., \& London, R. (1997). Brief physician advice for problem alcohol drinkers: A randomized controlled trial in community-based primary care practices. The fournal of the American Medical Association, 277, 1039-1045.

Gairin, I., House, A., \& Owens, D. (2003). Attendance at the accident and emergency department in the year before suicide: Retrospective study. The British fournal of Psychiatry, 83, $28-33$.

Healy, D. J., Barry, K., Blow, F., Welsh, D., \& Milner, K. K. (2006). Routine use of the Beck Scale for Suicide Ideation in a psychiatric emergency department. General Hospital Psychiatry, 28, 323-329.

Ilgen, M. A., Harris, A.H.S., Moos, R. H., \& Tiet, Q. Q. (2007). Predictors of a suicide attempt one year after entry into substance use disorder treatment. Alcobolism: Clinical \& Experimental Research, 31, 635-642.

Institute of Medicine. (2002). Reducing suicide: A national imperative. Washington, DC: National Academies Press.

Kapur, N., Cooper, J., Hiroeh, U., May, C., Appleby, L., \& House, A. (2004). Emergency department management and outcome for selfpoisoning: A cohort study. General Hospital Psychiatry, 26, 36-41.

Kessleer, R. C., Berglund, P., Borges, G., Nock, M., \& WANG, P. S. (2005). Trends in suicide ideation, plans, gestures, and attempts in the United States, 1990-1992 to 2001-2003. The Fournal of the American Medical Association, 293, 2487-2495.

Kessler, R. C., Borges, G., \& Walters, E. E. (1999). Prevalence of and risk factors for lifetime suicide attempts in the National Comorbidity Survey. Archives of General Psychiatry, 56, 617-626.

Kroenke, K., Spitzer, R. L., \& Williams, J. B. (2001). The PHQ-9: Validity of a brief depression severity measure. Fournal of General Internal Medicine, 19, 606-613.

Löwe, B., Spitzer, R. L., GräFe, K., Kroenke, K., Quenter, A., Zipfel, S., et al.
(2004). Comparative validity of three screening questionnaires for $D S M-I V$ depressive disorders and physicians' diagnoses. Fournal of Affective Disorders, 78, 131-140.

Lynskey, M. T., Glowinski, A. L., Todorov, A. A., Bucholz, K. K., Madden, P. A., Nelson, E. C., et al. (2004). Major depressive disorder, suicidal ideation, and suicide attempt in twins discordant for cannabis dependence and early-onset cannabis use. Archives of General Psychiatry, 61, 1026-1032.

McCaig, L., \& Burt, C. (2003). National Hospital Ambulatory Medical Care Survey: 2000 emergency department summary. Atlanta, GA: Centers for Disease Control.

Saunders, J. B., Aasland, O. G., Babor, T. F., Fuente, J. R., \& Grant, M. (1993). Development of the Alcohol Use Disorders Identification Test (AUDIT): WHO collaborative project on early detection of persons with harmful alcohol consumption-II. Addiction, 88, 791-804.

Smith, G. R., Burnam, M. A., Mosley, C. L., Hollenberg, J. A., Mancino, M., \& Grimes, W. (2006). Reliability and validity of the substance abuse outcomes module. Psychiatric Services, 57, 1452-1460.

Spitzer, R., Kroenke, K., \& Williams, J. (1999). Validation and utility of a self-report version of PRIME-MD: The PHQ primary care study. Primary care evaluation of mental disorders: Patient Health Questionnaire. The Fournal of the American Medical Association, 282, 1737-1744.

Wallace, P., Cutler, S., \& Haines, A. (1988). Randomized controlled trial of general practitioner intervention in patients with excessive alcohol consumption. British Medical Fournal, 297, 663-668.

Ware, J. E. (1976). Scales for measuring general health perceptions. Health Services Research, 11, 396-415.

Ware, J., Kosinski, M., \& Keller, S. D. (1996). A 12-item short-form health survey: Construction of scales and preliminary tests of reliability and validity. Medical Care, 34, 220-233.

Ware, J. E., \& Sherbourne, C. D. (1992). The MOS 36-item short-form health survey (SF36): I. Conceptual framework and item selection. Medical Care, 30, 473-483.

Manuscript Received: April 16, 2008

Revision Accepted: August 2, 2008 